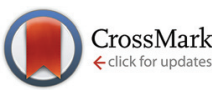

Cite this: Polym. Chem., 2016, 7, 7039

Received 12th October 2016, Accepted 29th October 2016

DOI: 10.1039/c6py01783e

www.rsc.org/polymers

\section{Poly(ester amide)s: recent insights into synthesis, stability and biomedical applications}

\begin{abstract}
Malte Winnacker* and Bernhard Rieger
Poly(ester amide)s (PEAs) are very important synthetic polymers with applications in many fields. The combination of the thermal and mechanical properties of polyamides with the biocompatibility and biodegradability of polyesters affords biomaterials of great interest especially for tissue engineering and drug delivery. Recent advances are elucidated herein with focus on synthesis and applications.
\end{abstract}

\section{Introduction}

Polyamides (PAs) and polyesters (PEs) are two of the most important polymer classes. ${ }^{1,2}$ PAs (monomers are connected via amide bonds) were established in the 1930's with Nylon 6,6 and Nylon 6, and meanwhile a variety of fossil- and bio-based polyamides exist for applications in many fields (commodities, automotive, biomedical). ${ }^{3}$ Polyesters (monomers are connected via ester bonds) are frequently used as mass plastics for consumables, and meanwhile also for special biomaterials in

WACKER-Lehrstuhl für Makromolekulare Chemie, Technische Universität München, Lichtenbergstraße 4, 85747 Garching bei München, Germany.

E-mail: malte.winnacker@makro.ch.tum.de

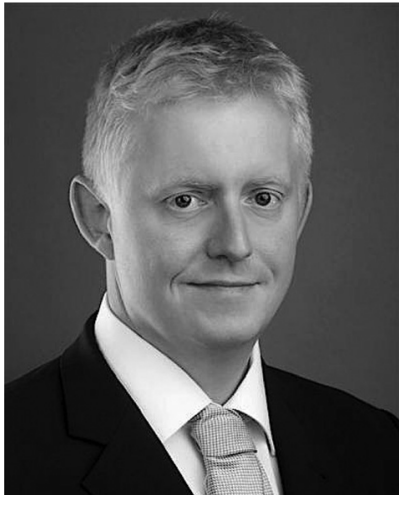

Malte Winnacker
Dr Malte Winnacker received his diploma (chemistry) at the University of Würzburg in 2006 and his PhD at the LudwigMaximilians-Universität München in 2010 under the direction of Prof. T. Carell. Then he served as a postdoctoral DAAD fellow at Stanford University with Prof. Eric T. Kool. Currently, he is a scientific coworker and Habilitand at the WACKER-Chair of Macromolecular Chemistry at Technische Universität München. His main research interests are sustainable polyamides and polyesters, as well as biomolecule-polymer conjugates. medicine due to their good mechanical properties, ability to hydrolyze and biocompatibility. ${ }^{1,2}$

Poly(ester amide)s (PEAs) are prominent polymers, which can combine the stiffness and the excellent thermal and mechanical properties of polyamides with the biocompatibility and biodegradability of polyesters. ${ }^{4,5}$ They have been investigated and applied as biodegradable plastics for consumables (Bayer, tradename BAK®). ${ }^{6}$ The first PEAs were synthesized in 1932 by Carothers from diacids, diols and diamines. ${ }^{7}$ Due to these properties, PEAs are an emerging group of very interesting polymers, ${ }^{8}$ which have gained an increasing impact within the past few years, especially for medical applications in drug delivery systems, hydrogels, non-viral gene carriers, smart materials, composites and adhesives and especially as scaffolds for tissue

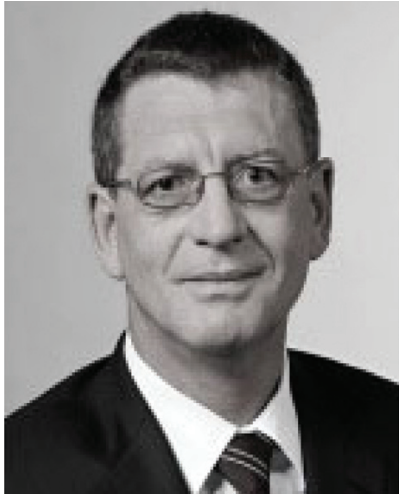

Bernhard Rieger
Prof. Dr Bernhard Rieger studied chemistry at the LudwigMaximilians-Universität München (LMU) and received his $P h D$ in 1988. After research at the University of Massachusetts at Amherst and in the plastics laboratory of BASF SE, he received his Habilitation in 1995 at the University of Tübingen. In the same year, he became professor ordinarius at the Department of Materials and Catalysis at the University of Ulm. In 2006, he was appointed as professor at TU München. Since then, he has been holding the WACKER-Chair of Macromolecular Chemistry and working as the director of the Institute of Silicon Chemistry at TUM. He has received the Philip-Morris Award (2006) and the Wöhler-Award for Sustainable Chemistry (2013). 
engineering (TE). ${ }^{9,10}$ Many synthetic polymers have been described for medical applications, e.g. for implants, drug delivery or $\mathrm{TE}^{11-14}$ and though also metals, ceramics or glasses can be used as TE scaffolds, polymers have meanwhile gained most attention for this due to their versatility of properties, which provides a basis for replacement, restoration and regeneration of tissue structure and/or function. Based on their structures, the designable alteration of hard and soft segments and a tunable amide bond/ester bond ratio, PEAs are very interesting in particular for suchlike applications. Many parameters like this ratio and also the microstructures play a role for their stability and degradation behavior. ${ }^{9}$ If the ester content is high, the degradability is favored, and if the amide content is high, the stability against degradation as well as thermal stability are the predominant properties. ${ }^{5}$ A lower degradation rate and lower $\mathrm{pH}$ drop compared to polyesters are additional important features of PEAs in view of their medical applications. This article describes the basic concepts of PEA synthesis and applications with focus on recent advancements.

\section{General aspects of poly(ester amide) synthesis}

PEAs can be synthesized via ring-opening polymerization (ROP) of cyclic monomers or via polycondensation of linear monomers. For instance, copolymers of $\alpha$-hydroxy acids and $\alpha$-amino acids, polydepsipeptides (PDPs), can be obtained by polymerization of morpholino-2,5-diones (synthesized by cyclization of amino acid derivatives) with different catalysts (e.g. SnOct $_{2}$ ) and enzymes (lipases) (Scheme 1A). ${ }^{9}$ Furthermore, copolymerization of lactams and lactones via ROP can afford different PEAs (Scheme 1B). Polycondensation can further be classified into melt polycondensation, interfacial polymerization, solution polycondensation and solid/ liquefied state polycondensation. Thus, depending on the exact procedures and the exact monomers used, regular, segmented or random PEAs can be synthesized - the respective reaction design can be complex, and the corresponding details have been reviewed elsewhere in detail. ${ }^{10}$ In brief, regular PEAs can e.g. be synthesized by thermal polycondensation of a diol and a diamide-diester (Scheme 1C), while segmented PEAs are preferably prepared by reaction of diesters with diamide diol units (Scheme 1D). A "classical" way to obtain random PEAs is a thermal polycondensation of diols, dicarboxylic acids and amino acids (Scheme 1E). PEAs can also be afforded by e.g. polycondensation of diamide-diols with a dicarboxylic acid (dichloride) (Scheme 1F) or via reaction of chloro acetate with amino acids (via metal halide salt; Scheme 1G). ${ }^{10}$ Furthermore, PEAs can be obtained by other solution polymerizations via reaction of an activated dicarboxylic acid (e.g. p-nitrophenol as the activating group) with an ester diamine salt or a diester diamine salt (e.g. with pTSA; Scheme $1 \mathrm{H}) \cdot{ }^{15,16}$ In a similar, further developed strategy for PDP synthesis, the $p$-toluene sulfonic acid salt of

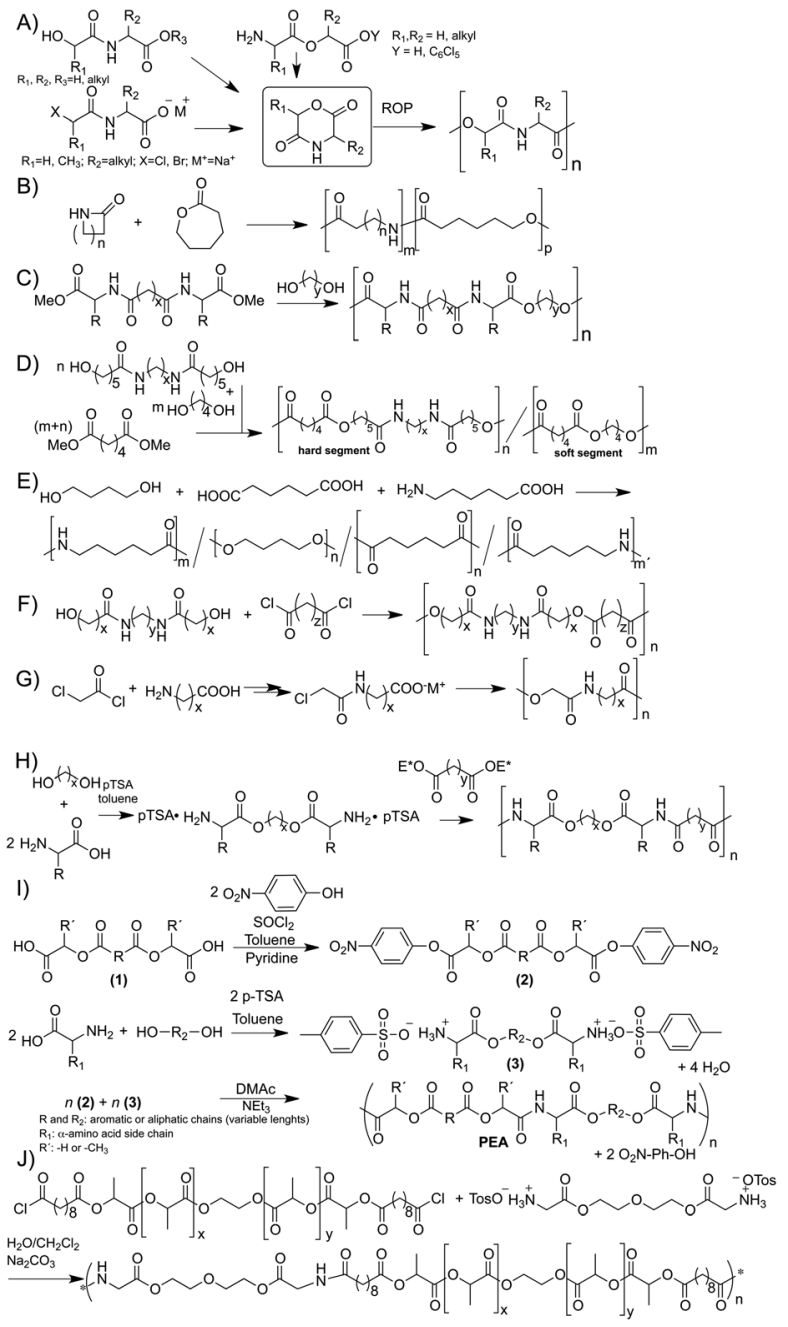

Scheme 1 Synthesis of polyesteramides (PEAs). (A) ROP of morpholino-2,5-diones; (B) ROP of lactams and lactones; (C) polycondensation of diol and diamide diester; (D) of diesters and diamide diols; (E) of diols, diacids and amino acids; (F) of diamide diol and dicarboxylic acid (dichloride); (G) polycondensation of a salt from chloroacetate derivatives of $\omega$-amino acid; $(H)$ of diester diamine salt with activated dicarboxylic acid; (I) of BAAD salt and nitrophenyl esters; (J) of $p$-toluenesulfonic salts of $\alpha$-amino acid derivatives and macrodiacyl chlorides from $\alpha$-hydroxy acids.

bis- $\alpha$-(L-amino acid)- $\alpha, \omega$-alkylene diesters (BAAD) is made to react with nitrophenyl esters derived from $\alpha$-hydroxy acids. The latter can be obtained in two steps, the reaction of an $\alpha$-hydroxy acid with a diacyl chloride, affording a dicarboxylic acid (1), and reaction of this acid with $p$-nitrophenol (in the presence of thionyl chloride and pyridine) (Scheme 1I). ${ }^{17}$ Another important strategy to obtain PEAs is the interfacial polycondensation of $p$-toluenesulfonic salts of $\alpha$-amino acids (or derivatives) and macrodiacyl chlorides from $\alpha$-hydroxy acids (Scheme 1J). ${ }^{18-20}$ Also routes to hyperbranched PEAs $^{21,22}$ as well as "mixed" procedures are possible (e.g. opening of a cyclic monomer, followed by a polycondensation; see later). ${ }^{10}$ 


\section{Recent progress in poly(ester amide)s based on amino acids}

Structure variety, biocompatibility, functionalities and stereoinformation of $\alpha$-amino acids, which are in nature building blocks for peptides and proteins, make these components highly interesting for biomaterials and thus for PEAs. ${ }^{23,24}$ The obtained materials can thus - to some extent - also be described as bioinspired. Many suchlike studies have been described and reviewed elsewhere in detail, ${ }^{8-10}$ and recent advances are elucidated here in brief. For instance, electrospun PEA with the incorporated nitroxyl radical, which is a highly reactive free radical with expanded biological functions, has been prepared. ${ }^{25}$ Here, polycondensation of di-p-nitrophenyl sebacate and $p$-toluenesulfonic acid salt of L-phenylalanine butane-1,4 diester afforded the PEA ("8-Phe-4"), which was then mixed with a nitroxyl oxide agent (4-amino-TEMPO). Electrospinning resulted in amorphous fibers, which showed biodegradation and changed with time into films. The drug was released in a controlled manner, making these fibers interesting for tissue engineering applications. Furthermore, PEAs based on alanin, 1,12-dodecanediol and sebacic acid were prepared and loaded with antimicrobial agents $\mathrm{AgNO}_{3}$ and chlorhexidine. ${ }^{26}$ Electrospinning resulted in fibers with different degrees of crystallinity, which had a great effect on the release of the agents, but which was not affected by the drugs. Both Gram negative (E. coli) and Gram positive (Micrococcus luteus) bacteria showed significant - and different - sensitivity to the inhibition of adhesion on the drug-loaded scaffolds. Biocompatibility of the scaffolds was proven by MTT assay, cell adhesion, proliferation and normal extension of monolayers of MDCK cells and HEp-2 cells.

Higher molecular weight, $\alpha$-amino acids containing PEAs with enhanced viscoelasticity (thus mimicking the extracellular matrix) for application in vascular tissue engineering were prepared by both interfacial and solution polymerization. ${ }^{27}$ They are thermally stable (decomposition temperature exceeds $300{ }^{\circ} \mathrm{C}$ ), which enables processing without degradation and change of MW. Nonfunctional PEA supported cell growth (human coronary artery smooth muscle cells (HCASMC)) and survival, whereas cell proliferation on functional PEAs was significantly lower than on tissue culture polystyrene (TCPS) for comparison. Further recent examples are cationic PEAs ${ }^{28}$ (in water soluble or insoluble variant), which can be used for attachment and proliferation of bovine aortic endothelial cells (BAECs), ${ }^{29}$ and PEAs with both aromatic and aliphatic side chains for vascular tissue engineering applications. ${ }^{30}$ In another study, an implantable miniinfusion type pump based on protein analog PEA based electrospun fibers was prepared for the controlled delivery of Fibroblast Growth Factor-9 (FGF9) (Phe-based, Fig. 1A). ${ }^{31}$ Loaded PEA fibers showed controlled FGF9 release over 28 days (limited burst effect) while maintaining FGF9 bioactivity. Proliferation of fibroblasts was supported by both FGF9-loaded and unloaded fibers for five days even under serum-depleted conditions. Cells on FGF9-

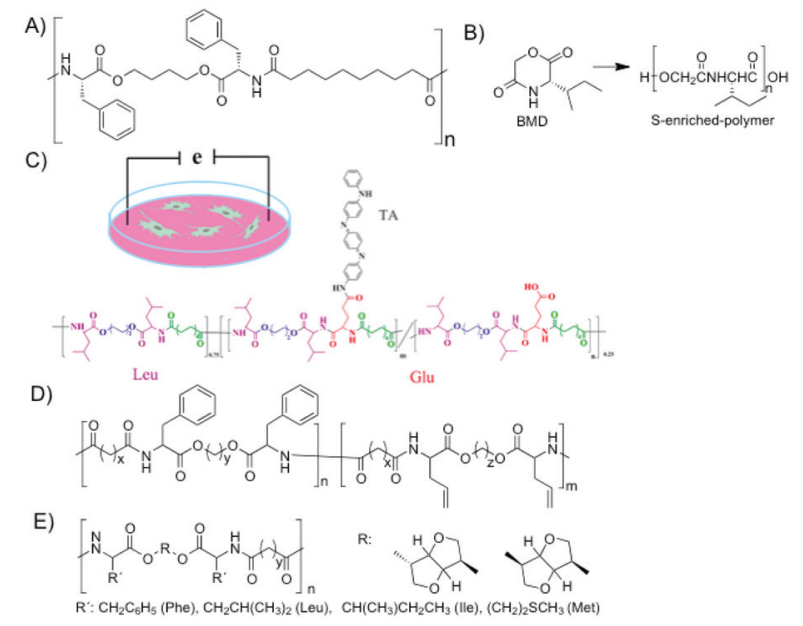

Fig. 1 PEAs based on (A) L-Phe; (B) on BMD and (C) tetraaniline-grafted PEAs based on Leu and Glu. Reprinted with permission from ref. 33. Copyright (2012) American Chemical Society. (D) PEAs with pendant $\mathrm{C}-\mathrm{C}$ double bonds; (E) PEAs derived from amino acids and dianhydrohexitols.

supplemented PEA mats showed upregulation of PDGFR $\beta$ dependent on the concentration and cell type. ${ }^{31}$ With regard to morpholino-2,5-dione polymerization (see section 2), lipasecatalyzed ROP of isoleucine-based 3(S)-sec-butylmorpholine2,5-dione (BMD) was described to afford poly(BMD), an optically active PEA with specific stereoregularity (dyads) (Fig. 1B). ${ }^{32}$ Different lipases and parameters (enzyme concentration, time, temperature) were screened, and conversions up to $70 \%$ and molecular weights from 5500-10700 were obtained. It was found that water content is an important factor for the control of both conversion and molecular weight: the increasing water content results in enhanced polymerization rates while the MW of poly(BMD) decreases.

Tetraaniline grafted PEAs (PEA- $g$-TA) based on Leu and Glu were also synthesized and characterized (Fig. 1C). ${ }^{33}$ They exhibited good electroactivity, mechanical properties and biodegradability, are nontoxic and provide good adhesion and proliferation of mouse preosteoblastic MC3T3-E1 cells. These copolymers stimulated by the pulsed electrical signal could serve to promote the differentiation of MC3T3-E1 cells in comparison to TCPs. Based on this electrical stimulation directly to the desired area, their potential in favor of long-time application in vivo as a bone repair scaffold material in TE could be shown.

Double-bonds enable further functionalization of the PEAs and have been described as additional groups for several monomer types. Important examples are L-Phe and D,L-2-allylglycine based PAs with pendant $\mathrm{C}-\mathrm{C}$ double bonds for subsequent carboxylic acid, amino and sulfonate functionalization (Fig. 1D). ${ }^{34}$ This strategy enables the preparation of biodegradable functional PEA-based hydrogels. ${ }^{35}$ Furthermore, dianhydrohexitols can be used as secondary diols to react with $\alpha$-amino acids to afford PEAs with incorporated hexitol-units and pendant residues (Fig. 1E). ${ }^{36}$ 
Unsaturated PEAs based on other compounds have also been described (which can also be further functionalized via their double bond; see later). ${ }^{9}$ Sequential PEAs derived from glycine, diols and dicarboxylic acids were also synthesized by a two-step method that involved a final thermal polyesterification. ${ }^{37}$ Similar PEAs were prepared from L-lysine as the diamine unit. The possibility of linking pharmacologically active compounds to the carboxylic groups of lysine makes these materials even more interesting e.g. for drug delivery systems. ${ }^{38}$ Phe- and Lys-based PEAs were also investigated in terms of endothelial cell adhesion and inflammation in vitro, ${ }^{39}$ and arginine-based PEAs have been used as non-viral-gene delivery reagents. ${ }^{40}$ PEAs based on the chloroacetate derivatives of an $\omega$-amino acid or a diamine are similar examples. ${ }^{41,42}$ With regard to blood and the cellular in vitro response, it was shown that monocytes adherent to leucine and lysine based PEAs secreted reduced levels of the proinflammatory interleucines (IL)- 6 and IL-1b into the culture supernatant in comparison to other polymers, but secreted higher amounts of IL-1 receptor antagonist, an anti-inflammatory mediator. ${ }^{43}$ Versatile methods for studying the enzymatic degradation of suchlike PEAs have also been developed. ${ }^{44}$ Another very interesting example in this context is lysine-based PEA microspheres for controlled drug delivery (lipophilic drug dexamethasone) in ophthalmology. ${ }^{45}$ Their potential could be further proved by monitoring the location of the microspheres in rat eyes. Similar derivatives have already been studied by DSM Biomedical in preclinical evaluations and are meanwhile also used clinically in cardiovascular application. ${ }^{46-48}$ The biocompatibility of similar PEA microfibrils in ocular tissue could also be demonstrated. ${ }^{49}$

\section{Poly(ester amide)s based on $\varepsilon$-caprolactone and $\varepsilon$-caprolactam}

Cyclic monomers $\varepsilon$-caprolactam (CLa) and $\varepsilon$-caprolactone (CLo) belong to the most relevant representatives of the lactone and, respectively, the lactam family. Therefore, their copolymerization to PEAs by means of ROP has been intensively studied, and many procedures have been used for the preparation of $\mathrm{P}(\mathrm{CLO}-\mathrm{Co}-\mathrm{CLa})$. Early studies described the copolymerization of CLa, $\alpha$-pyrrolidone (PL) or $\alpha$-piperidone (PP) with CLo or $\delta$-valerolactone (VLo) in the presence of an alkali metal. ${ }^{50}$ For copolymerization of CLa or PL with CLo and PL with VLo, conversions of above $70 \%$ and reduced viscosities of about 0.5 (in $m$-cresol) at $35{ }^{\circ} \mathrm{C}$ for all compositions were obtained. Copolymerization of CLa with VL at $180{ }^{\circ} \mathrm{C}$, a conversion of $25 \%$ and a reduced viscosity of 0.5 were obtained, but the polymer polycaproamide was formed, while at $90{ }^{\circ} \mathrm{C}$ the conversion was $50 \%$ but the polymer was polyvalerolactone. Copolymerization of PP with CL or VL resulted only in a polyester. The proposed mechanism involves chain propagation by the stepwise addition of the lactam at the end of the chain and by stepwise addition of the lactone at the other end of the chain, resulting effectively in the formation of polyester and polyamide blocks/segments. The results obtained through a reaction with alkali metal salt of the lactam, IR data, elemental analysis and determination of physical properties of these copolymers supported this mechanism. Anionic copolymerization of CLa and CLo has also been described with $\varepsilon$-caprolactam magnesium bromide as the initiator. ${ }^{51}$ Polymers with 5-25 wt\% CLo were obtained. It was possible to conduct polymerization at $110{ }^{\circ} \mathrm{C}$. CLo-content and temperature influenced the mechanical properties, and it was not possible to restrict exchange transacylation reactions, which progress during polymerization, by kinetic tools. Furthermore, two types of CLO-CLa copolymers were prepared by catalyzed hydrolytic ROP (Scheme 2). ${ }^{52}$ First, both cyclic comonomers were simultaneously in the reaction medium, where copolymers with random distribution were afforded (as evidenced by NMR). For the second type, cyclic comonomers were added sequentially, yielding diblock PEAs. Polymers were analyzed by DSC, X-ray scattering (SAXS and WAXS), TEM and SEM, and biodegradation was also studied. Interestingly, in a wide composition range only the CLa units were capable of crystallization. Comparison between block and random copolymers gave a good opportunity to distinguish the dilution effect of CLO units on the crystallization and melting of the polyamide phase from the chemical composition effect for random copolymers, which makes the crystallization of the polymer strongly dependent on composition. Degradation in composted soil showed that much faster degradation was obtained for random PEAs with a CLo content larger than 30\% than for neat PCL (synergistic effect).

Further biodegradation studies were conducted under the attack of yeast Cryptococcus laurentii at $20{ }^{\circ} \mathrm{C} .{ }^{53} \mathrm{CLO}-\mathrm{CLa}$ copolymers as well as copolymers prepared by a two-step polycondensation reaction of hexanediol-1,6, hexanediamine-1,6 and adipoyl chloride were investigated. Under biotic conditions, these copolymers were found to be readily degradable, which was shown by weight loss, GPC, NMR and tensile measurements. Biodegradation occurred much faster under milder conditions compared to abiotic hydrolysis. The enzymatic hydrolysis of ester groups into acids and hydroxyl groups was proved by NMR, while no breakdown of amide bonds was observed under the biotic conditions. Suchlike copolymers were recently studied in a wide composition range and in the wet and dry states. Crystalline and amorphous phases of these PEAs were characterized, the first by wide-angle X-ray scattering and the latter by the thermally stimulated depolarization current technique. ${ }^{54}$ A microwave synthesis of PCLa-PLCo has

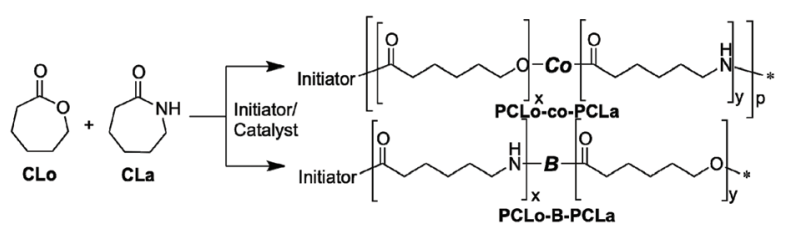

Scheme 2 Copolymerization of CLo and CLa for random and block PEAs. ${ }^{52}$ 
also been described. ${ }^{55}$ Here, a variable frequency furnace, programmed to a set temperature and controlled by a pulsed power on-off system was used to induce anionic copolymerization of these monomers, which showed effective adsorption of microwave energy. Dielectric properties were measured in a range of $0.4-3 \mathrm{GHz}$, and the polymer yields were $70 \%$. In comparison with conventional thermal and microwave copolymerization studies, it was thus shown that an effective microwave method for CLa/CLo copolymerization was developed with higher yield, higher amide content and higher $T_{\mathrm{g}}$ 's relative to the thermal process. Further studies describe similar anionic ROP of these cyclic monomers as well as their thermoanalytical data in detail. ${ }^{56-58}$

\section{Polyesteramides derived from various linear diacids, diamines and diols}

Copolymers of CLa, 1,4-butanediol and adipic acid possess interesting mechanical properties and are suitable for different applications. These polymers have for instance been prepared in a one-batch two-step reaction and manufactured as nonwoven 3D textile scaffolds applicable for tissue engineering scaffolds (Scheme 3A). ${ }^{59}$ Structural conformity of different batches was confirmed by NMR and SEC. Production was successfully performed via simultaneous ROP of CLa and polycondensation with 1,4-butanediol and adipic acid at $250{ }^{\circ} \mathrm{C}$ under high vacuum, and Soxhlet extraction allowed optimal cleaning. The effect of scaffold extraction before cell seeding was analyzed by cytotoxicity tests and XPS. The carriers were then seeded with human preadipocytes and examined for cellular proliferation and differentiation, which was shown to be very effective. This makes this kind of PEA suitable for clinical use.

Novel PEAs derived from 1,4-butanediol, dimethyl adipate and a preformed $\alpha, \omega$-amino alcohol from aminobutane and

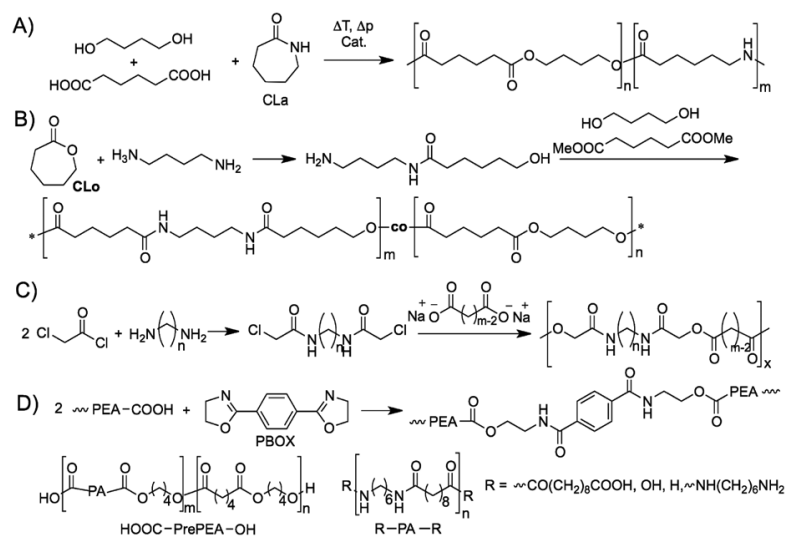

Scheme 3 PEA synthesis (A) from adipic acid, butanediol and CLa; (B) from TMDA, CLo and adipic acid; (C) based on glycolic acid (dichloride) and diamines; (D) from sebacic acid and PBOX. caprolactone were also prepared and electrospun from solution (Scheme 3B). ${ }^{60}$ Many effects on fibre morphology and diameter were investigated, namely by increasing the ratio of amide/ ester groups in the copolymer, polymer concentration, solvent mixtures and applied voltage. The obtained fibres were randomly oriented. Increasing amide concentration increased the fiber quality and homogeneity, and the solvent mixture $\mathrm{CHCl}_{3}$ / $\mathrm{HCOOH}$ gave the best electrospinning results. The fibers were characterized by SEM, DSC and FT-IR, showing that they are amorphous compared to the pristine samples. These fibers are thus potential candidates for applications as tissue engineering scaffolds. Aliphatic PEAs with a periodic sequential structure consisting of ester and amide bonds were also synthesized by a two-step polycondensation reaction of adipate, butane-1,4diamine and linear diols (3-6 methylene units). ${ }^{61}$

Similarly, PEAs derived from glycolic acid (respectively its dichloride), 1,6-hexanediamine and even aliphatic dicarboxylic acids (adipic acid and dodecanedioic acid) have been synthesized and analyzed (Scheme 3C). ${ }^{62}$ Isothermal crystallization from diol and glycerin solutions resulted in chain-folded lamellar crystals, and the crystalline habit was investigated by real space electron microscopy. Triclinic and monoclinic unit cells were obtained, in which the crystallographic parameter was close to the typical distance between hydrogen-bonded chains. The molecular conformation of both semicrystalline polymers deviates from the all-trans conformation typical of aliphatic PAs and PEs with a large number of methylene groups. In another study, polycondensation of a bio-based sebacic acid, 1,4-butanediol and introduction of 1,4-butanediamine as a comonomer afforded different interesting PEAs. ${ }^{63}$ The homopolymer was semicrystalline with a $T_{\mathrm{m}}$ of $65^{\circ} \mathrm{C}$. An amide content over $10 \%$ resulted in two melting endotherms and a significant decrease of their melting enthalpies. The crystallinity decrease with increasing amide content was shown by the WAXD results, and FT-IR measurements confirmed intramolecular as well as intermolecular hydrogen bonding, as well as the fact that the area of the amide-amide hydrogen bonded $\mathrm{NH}$ band increases during hydrolysis, while the area of the amide-ester hydrogen bonded $\mathrm{NH}$ band decreases. Furthermore, the degradability of the PEA series increased with the amide content. In other work, the Nylon 610 oligomer (PrePA) was prepared from the reaction of Nylon610 salt with sebacic acid. Polyester prepolymers (PrePEAs) with an amide content from 10-60 mol\% were then synthesized via melt polycondensation from adipic acid, 1,4butanediol and the PrePA with stannous chloride as catalysts. Chain extension of these PrePEAs was then carried out using 2,2'-(1,4-phenylene)-bis(2-oxazoline) (PBOX) and adipoyl biscaprolactamate as combined chain extenders, resulting in extended biodegradable PEAs (ExtPEAs) with $T_{\mathrm{m}}$ from 95.2 to $156{ }^{\circ} \mathrm{C}$, the initial decomposition temperature over $325.3{ }^{\circ} \mathrm{C}$ and the tensile strength up to $33.1 \mathrm{MPa}$ (Scheme 3D). ${ }^{64}$

Regarding (renewable) sebacic acid, poly(1,3-diamino-2hydroxypropane-co-polyol sebacate) (APS) elastomers have also been synthesized and used for the fabrication of airway stents. ${ }^{65}$ In contrast to airway stents that would have to be sur- 
gically removed after a certain time, these APS-based stents are biodegradable with high strength, flexibility and transparency. Driven by the motivation that, to date, other stents have involved inherent risks such as trauma during surgical stent removal, infection and sedation problems, microfabrication technology with bioresorbable polymers was thus combined with the goal of a stent ultimately capable of improving patient safety and treatment outcomes. 1:2 APS and 2:1 APS were synthesized, maintaining the ratio of 1,3-diamino-2hydroxy-propane (DAHP) and glycerol as $1: 2$ and $2: 1$, respectively. APS was synthesized via step-growth polymerization, and stents with nano-gratings in the size 500, 750 and $1000 \mathrm{~nm}$ were created via print molding. The 1:2 stent exhibited desired mechanical properties (Young's Moduli approx. 2 $\mathrm{MPa}$ ) after $24 \mathrm{~h}$ of curing (optimum) - a value similar to existing silicone stents. ${ }^{65}$

Random multiblock PEAs containing poly(L-lactide) (PLLA) and cycloaliphatic amide segments were also synthesized from the telechelic oligomer of $\alpha, \omega$-hydroxyl terminated PLLA, 1,3cyclohexylbis(methylamine), and sebacoylchloride by a twostep interfacial polycondensation method. ${ }^{66}$ It was shown that PEAs having a lower content of PLLA (PEA1 and PEA2) have a crystallization pattern analogous to polyamides, whereas PEA 3 (higher content of PLLA) showed two crystalline phases characterized by polyester and polyamide segments. PEA 3 had a higher degradation rate than PEA 1 and PEA 2, as determined by biodegradation studies with enzyme lipase from Candida cylindracea. PEAs constituted by glycolic acid, adipic acid ratios of 1,3-pentane-diamine and 1,5-pentanediamine units were also studied and showed high dependence of physical properties on the final composition, e.g. an increase in degradability with an increasing amount of 1,3 pentanediamine. ${ }^{67}$

\section{Unsaturated polyesteramides}

PEAs with unsaturated amino acid residues have been described in section 3. As another example, PEAs with positively charged guanidine groups and pendant $\mathrm{C}-\mathrm{C}$ double bonds were also prepared. ${ }^{68}$ Furthermore, unsaturated PEAs based on ethylene glycol lactate diol, maleic anhydride and toluene-2,4-diisocyanate afford a crosslinked resin with unsaturated double bonds, which is very interesting for biomaterials due to its porous structure (formation of $\mathrm{CO}_{2}$ during synthesis), flexibility (resulting in shape-memory characteristics) and a $T_{\mathrm{g}}$ close to human body temperature (Fig. 2). ${ }^{69}$

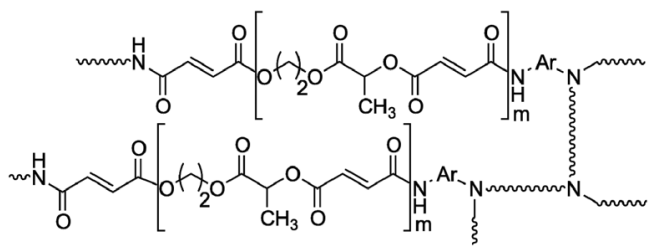

Fig. 2 Unsaturated PEAs, crosslinked, containing lactic acid units.

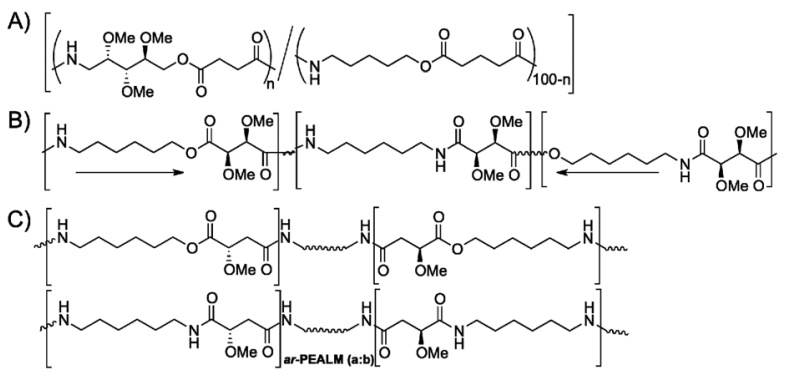

Fig. 3 PEAs derived from (A) L-arabinose; (B) tartaric acid and (C) malic acid.

\section{Polyesteramides from other natural products}

In addition to the amino acid and sebacic acid derived PEAs described above, several other natural product based PEAs have been described. Examples are carbohydrate-derived, stereocenter-containing PEAs from L-arabinose, succinyl and glutaryl moieties (Fig. 3A). ${ }^{70}$ For those, the degradation rate could be enhanced by increasing the amount of the sugarbased monomer. Biodegradable PEAs from tartartic acid have also been investigated $(M>80000)$, which showed $T_{\mathrm{m}}$ and $T_{\mathrm{g}}$ values ranging from 100 to 230 and 50 to $100{ }^{\circ} \mathrm{C}$, respectively (Fig. 3B). ${ }^{71}$ Similarly, PEAs derived from L-malic acid were prepared with ester to amide group ratios from 1:50 up to $1: 2$, with $O$-methyl-L-malic acid, 1,6-hexanediol and 1,6 hexanediamine as the comonomers (Fig. 3C). The ester linkage was incorporated using 6-aminohexyl-pentachlorophenyl $O$-methyl-L-malate as the comonomer in the polycondensation of bis(pentachlorophenyl)-O-methyl-L-malate with 1,6-hexanediamine. $^{72}$ Isoregic ir-PEALM $(1: 1)$ and aregic ar-PEALM were obtained. The molecular weight of PEALM is between 10000 and 50000 . The $T_{\mathrm{m}}$ of these PEAs decreased with the content of ester groups from 168 to $144{ }^{\circ} \mathrm{C}$, and $T_{\mathrm{g}}$ falling from 60 to $10{ }^{\circ} \mathrm{C}$. Thermal decomposition of PEALM initiated around $260{ }^{\circ} \mathrm{C}$. Degradation occurred in water at $\mathrm{pH} 7.4,37{ }^{\circ} \mathrm{C}$, at a rate that increased with the content of ester groups.

\section{Conclusion}

Polyesteramides are very important biodegradable polymers, which show some improved properties compared to polyesters (e.g. strength) due to the presence of both ester and amide bonds. Many interesting studies refer to the synthesis and application of PEAs with a variety of different architectures, which can afford amorphous, semicrystalline and elastomeric materials. A lot of biomedical applications for these polymers are studied in terms of drug delivery, hydrogels or tissue engineering. Based on their promising property profiles, further effort and research will be spent for PEAs, which are anticipated to play a growing role as high performance 
materials in the future for general and special applications especially in the (bio)medical field.

\section{Notes and references}

1 K. Marchildon, Macromol. React. Eng., 2011, 5, 22-54.

2 A. C. Albertsson and I. K. Varma, Biomacromolecules, 2003, 4, 1466-1486.

3 M. Winnacker and B. Rieger, Macromol. Rapid Commun., 2016, 37, 1391-1413.

4 (a) R. Timmermann, Polyesteramides, in Biopolymers, ed. Y. Doi and A. Steinbüchel, Wiley-VCH, New York, 2003, vol. 4, pp. 315-328; (b) R. Timmermann, K. J. Idel, W. SchulzSchlitte and E. Grigat, WO 9942514, 1999.

5 A. Rodríguez-Galán, L. Franco and J. Puiggalí, Biodegradable Poly(ester amide)s: Synthesis and Applications, in Biodegradable Polymers: Processing, Degradation, and Applications, ed. G. P. Felton. Nova Science Publishers Inc, 2011.

6 S. Mecking, Angew. Chem., Int. Ed., 2004, 43, 1078-1085.

7 W. H. Carothers and J. W. Hill, J. Am. Chem. Soc., 1932, 54, 1566-1569.

8 A. C. Fonseca, M. H. Gil and P. N. Simões, Prog. Polym. Sci., 2014, 39, 1291-1311.

9 K. Ghosal, M. S. Latha and S. Thomas, Eur. Polym. J., 2014, 60, 58-68.

10 A. Rodriguez-Galan, L. Franco and J. Puiggali, Polymers, 2011, 3, 65-99.

11 B. D. Ulery, L. S. Nair and C. T. Laurencin, J. Polym. Sci., Part B: Polym. Phys., 2011, 49, 832-864.

12 K. A. Gilmore, M. W. Lampley, C. Boyer and E. Harth, Adv. Drug Delivery Rev., 2016, 98, 77-85.

13 M. Winnacker and B. Rieger, Polym. Int., 2016, DOI: 10.1002/pi.5261.

14 M. F. Maitz, Biosurface and Biotribology, 2015, 1, 161-176.

15 R. Katsarava, Macromol. Symp., 2003, 199, 419-429.

16 R. Katsarava, V. Beridze, N. Arabuli, D. Kharadze, C. C. Chu and C. Y. Won, J. Polym. Sci., Part A: Polym. Chem., 1999, 37, 391-407.

17 R. Katsarava, N. Ochkhikdze, D. Tugushi and Z. D. Gomurashvili, AABB-poly(depsipeptide) biodegradable polymers and methods of use, US 2010/0040664 A1, 2010.

18 A. C. Fonseca, A. C. Serra, J. F. J. Coelho, M. H. Gil and P. N. Simões, Polym. Int., 2013, 62, 736-743.

19 A. C. Fonseca, J. F. J. Coelho, M. H. Gil and P. N. Simões, Polym. Bull., 2014, 71, 3085-3109.

20 A. C. Fonseca, J. F. J. Coelho, J. F. A. Valente, T. R. Correia, I. J. Correia, M. H. Gil and P. N. Simões, J. Biomater. Sci., Polym. Ed., 2013, 24, 1391-1409.

21 P. Froehling, J. Polym. Sci., Part A: Polym. Chem., 2004, 42, 3110-3115.

22 S. Reven, J. Grdadolnik, J. Kristl and E. Žagar, Int. J. Pharm., 2010, 396, 119-126.
23 H. Sun, F. Meng, A. A. Dias, M. Hendriks, J. Feijen and Z. Zhong, Biomacromolecules, 2011, 12, 1937-1955.

24 C.-C. Chu, JFBI, 2012, 5:1, 1-31.

25 L. Li and C.-C. Chu, J. Biomater. Sci., Polym. Ed., 2009, 20, 341-361.

26 L. J. del Valle, M. Roa, A. Díaz, M. T. Casas, J. Puiggalí and A. Rodríguez-Galán, J. Polym. Res., 2012, 19, 9792.

27 D. K. Knight, E. R. Gillies and K. Mequanint, Biomacromolecules, 2011, 12, 2475-2487.

$28 \mathrm{~J} . \mathrm{Wu}, \mathrm{D} . \mathrm{Wu}, \mathrm{M}$. A. Mutschler and C.-C. Chu, Adv. Funct. Mater., 2012, 22, 3815-3823.

29 J. Wu and C.-C. Chu, J. Mater. Chem. B, 2013, 1, 353-360.

30 P. Karimi, A. S. Rizkalla and K. Mequanint, Materials, 2010, 3, 2346-2368.

31 S. S. Said, J. G. Pickering and K. Mequanint, Pharm. Res., 2014, 31, 3335-3347.

32 Y. Feng, D. Klee and H. Höcker, Macromol. Biosci., 2001, 1, 66-74.

33 H. Cui, Y. Liu, M. Deng, X. Pang, P. Zhang, X. Wang, X. Chen and Y. Wei, Biomacromolecules, 2012, 13, 2881-2889.

34 X. Pang and C.-C. Chu, Biomaterials, 2010, 31, 3745-3754.

35 X. Pang and C.-C. Chu, Polymer, 2010, 51, 4200-4210.

36 Z. Gomurashvili, H. R. Kricheldorf and R. Katsarava, J. Macromol. Sci., Part A: Pure Appl.Chem., 2000, 37, 215227.

37 L. Asín, E. Armelin, J. Montané, A. Rodríguez-Galán and J. Puiggalí, J. Polym. Sci., Part A: Polym. Chem., 2001, 39, 4283-4293.

38 M. Vera, L. Franco and J. Puiggalí, J. Polym. Sci., Part A: Polym. Chem., 2008, 46, 661-667.

39 J. A. Horwitz, K. M. Shum, J. C. Bodle, M. Deng, C.-C. Chu and C. A. Reinhart-King, J. Biomed. Mater. Res., Part A, 2010, 95, 371-380.

40 D. Yamanouchi, J. Wu, A. N. Lazar, K. C. Kent, C.-C. Chu and B. Liu, Biomaterials, 2008, 29, 3269-3277.

41 M. Vera, A. Rodríguez-Galán and J. Puiggalí, Macromol. Rapid Commun., 2004, 25, 812-817.

42 M. Vera, M. Admetlla, A. Rodríguez-Galán and J. Puiggali, Polym. Degrad. Stab., 2005, 89, 21-32.

43 K. M. DeFife, K. Grako, G. Cruz-Aranda, S. Price, R. Chantung, K. Macpherson, R. Khoshabeh, S. Gopalan and W. G. Turnell, J. Biomater. Sci., Polym. Ed., 2009, 20, 1495-1511.

44 A. Ghaffar, G. J. J. Draaisma, G. Mihov, P. J. Schoenmakers and Sj. Van der Wal, J. Chrom. A, 2013, 1286, 29-40.

45 V. Andrés-Guerrero, M. Zong, E. Ramsay, B. Rojas, S. Sarkhel, B. Gallego, R. de Hoz, A. I. Ramírez, J. J. Salazar, A. Triviño, J. M. Ramírez, E. M. del Amo, N. Cameron, B. de-las-Heras, A. Urtti, G. Mihov, A. Dias and R. HerreroVanrell, J. Controlled Release, 2015, 211, 105-117.

46 K. A. Messier, A. Dias, G. Mihov and B. Turnell, https:// www.dsm.com/content/dam/dsm/medical/en_US/documents/ pp-degradable-polyesteramides---a-new-platform-for-ophthalmic-drug-delivery.pdf, accessed October 2016.

47 http://www.dsm.com/markets/medical/en_US/products-page/ products-resorbable-materials.html, accessed October 2016. 
48 G. Mihov, G. Draaisma, A. Dias, B. Turnell and Z. Gomurashvili, J. Controlled Release, 2010, 148, e46-e47.

49 M. Kropp, K.-M. Morawa, G. Mihov, A. K. Salz, N. Harmening, A. Franken, A. Kemp, A. A. Dias, J. Thies, S. Johnen and G. Thumann, Polymers, 2014, 6, 243-260.

50 H. Komoto, Makromol. Chem., 1968, 115, 33-42.

51 A. Bernášková, D. Chromcová, J. Brožek and J. Roda, Polymer, 2004, 45, 2141-2148.

52 R. M. Michell, A. J. Müller, V. Castelletto, I. Hamley, G. Deshayes and P. Dubois, Macromolecules, 2009, 42, 6671-6681.

53 X. Chen, K. E. Gonsalves and J. A. Cameron, J. Appl. Polym. Sci., 1993, 50, 1999-2006.

54 D. Newman, E. Laredo, A. Bello and P. Dubois, Macromolecules, 2014, 47, 2471-2478.

55 X. Fang, R. Hutcheon and D. A. Scola, J. Polym. Sci., Part A: Polym. Chem., 2000, 38, 1379-1390.

56 M. Gupta, F. Siegmund, E. Haberstroh, M. Rosenthal, D. A. Ivanov, M. W. M. Fijten, X. Zhu and M. Möller, Macromol. Mater. Eng., 2014, 299, 1343-1351.

57 D. Chromcová, L. Baslerová, J. Roda and J. Brožek, Eur. Polym. J., 2008, 44, 1733-1742.

58 J. Merna, D. Chromcová, J. Brožek and J. Roda, Eur. Polym. J., 2006, 42, 1569-1580.

59 K. Hemmrich, J. Salber, M. Meersch, U. Wiesemann, T. Gries, N. Pallua and D. Klee, J. Mater. Sci.: Mater. Med., 2008, 19, 257-267.
60 P. Garg, D. Klee, H. Keul and M. Möller, Macromol. Mater. Eng., 2009, 294, 679-690.

61 H. Tetsuka, Y. Doi and H. Abe, Macromolecules, 2006, 39, 2875-2885.

62 M. T. Casas and J. Puiggalí, J. Polym. Sci., Part B: Polym. Phys., 2009, 47, 194-206.

63 S. Y. Lee, J. W. Park, Y. T. Yoo and S. S. Im, Polym. Degrad. Stab., 2002, 78, 63-71.

64 Y. Hao, M. Chen, J. Zhao, Z. Zhang and W. Yang, Ind. Eng. Chem. Res., 2013, 52, 6410-6421.

65 J. Wang, K. G. Boutin, O. Abdulhadi, L. D. Personnat, T. Shazly, R. Langer, C. L. Channick and J. T. Borenstein, Adv. Healthcare Mater., 2013, 2, 1329-1336.

66 G. E. Luckachan and C. K. S. Pillai, J. Polym. Sci., Part A: Polym. Chem., 2006, 44, 3250-3260.

67 S. K. Murase, L. Franco, L. J. del Valle and J. Puiggalí, J. Appl. Polym. Sci., 2014, 131, 40102.

68 X. Pang, J. Wu, C. Reinhart-King and C.-C. Chu, J. Polym. Sci., A: Polym. Chem., 2010, 48, 3758-3766.

69 C. Xiao and Y. He, Polym. Int., 2007, 56, 816-819.

70 I. Molina Pinilla, M. Bueno Martinez, F. Zamora Mata and J. A. Galbis, Macromolecules, 2002, 35, 2977-2984.

71 C. Regaño, A. Martínez de Ilarduya, J. I. Iribarren and S. Muñoz-Guerra, J. Polym. Sci., Part A: Polym. Chem., 2000, 38, 2687-2696.

72 C. Regaño, A. Alla, A. Martínez de Ilarduya and S. MuñozGuerra, Macromolecules, 2004, 37, 2067-2075. 